\title{
Fermi establishes classical novae as a distinct class of gamma-ray sources
}

\section{The Fermi-LAT Collaboration* $†$}

A classical nova results from runaway thermonuclear explosions on the surface of a white dwarf that accretes matter from a low-mass main-sequence stellar companion. In 2012 and 2013, three novae were detected in $\gamma$ rays and stood in contrast to the first $\gamma$-ray-detected nova V407 Cygni 2010, which belongs to a rare class of symbiotic binary systems. Despite likely differences in the compositions and masses of their white dwarf progenitors, the three classical novae are similarly characterized as soft-spectrum transient $\gamma$-ray sources detected over 2- to 3-week durations. The $\gamma$-ray detections point to unexpected high-energy particle acceleration processes linked to the mass ejection from thermonuclear explosions in an unanticipated class of Galactic $\gamma$-ray sources.

I he Fermi-LAT (Large Area Telescope) (I), launched in 2008, continuously scans the sky in $\gamma$ rays, thus enabling searches for transient sources. When a nova explodes in a symbiotic binary system, the ejecta from the white dwarf surface expand within the circumstellar wind of the red giant companion, and high-energy particles can be accelerated in a blast wave driven in the high-density environment (2) so that variable $\gamma$-ray emission can be produced,

${ }^{*}$ All authors with their affiliations appear at the end of this paper. †Corresponding author. E-mail: teddy.cheung@nrl.navy.mil (C. C. Cheung); pierre.jean@irap.omp.eu (P. Jean); shore@df. unipi.it (S. N. Shore) as was detected at $>100-\mathrm{MeV}$ energies by the LAT in V407 Cygni 2010 (V407 Cyg) (3). In a classical nova, by contrast, the ejecta quickly expand beyond the confines of the compact binary into a much lower-density environment. High-energy particle acceleration could therefore be related to a bow shock driven by the ejecta in the interstellar medium or to turbulence and eventually weaker internal shocks formed in the inhomogeneous ejecta itself. The contribution of such expanding nova shells to cosmic-ray acceleration had been considered (4), but no predictions have so far been made for $>100-\mathrm{MeV} \gamma$ rays. The classical novae (or simply "novae" where appropriate) detected by the LAT with statistical significance of 12 to $20 \sigma$ (Table 1 and Fig. 1)-V959 Monocerotis 2012 (V959 Mon), V1324 Scorpii 2012 (V1324 Sco), and V339 Delphini 2013 (V339 Del)were unanticipated. These observed $\gamma$ rays have higher energies than nuclear line emission by radioactive decay at $\sim \mathrm{MeV}$ energies that remain undetected in individual novae (5) and $\lesssim$ $0.1-\mathrm{MeV}$ emission detected in isolated cases (6).

V959 Mon was detected as a transient $\gamma$-ray source in June 2012 by the LAT while close $\left(\sim 20^{\circ}\right.$ separation) to the Sun (7) and then optically in August (8). Ultraviolet spectroscopy revealed an oxygen-neon nova (9), recognized as the class with the most massive white dwarfs $\left(\gtrsim 1.1 M_{\odot}\right)$ with massive ( $\gtrsim 8 M_{\odot}$ ) progenitors [e.g., (10)]. The expected peak visual magnitude of $\sim 5$ would have been observable with the naked eye $\sim 50$ days earlier, when the $\gamma$-ray transient was detected (9). V339 Del (11) was detected in August 2013 in a LAT-pointed observation triggered by its high optical brightness [4.3 magnitude at peak $(12,13)]$. Optical spectra of V339 Del suggest a carbon-oxygen nova (14), which are more common than the oxygen-neon types, with less massive white dwarfs evolved from $\lesssim 8 M_{\odot}$ mainsequence progenitors. Optical brightening of V1324. Sco was detected in May 2012 (15) and found in LAT $\gamma$-ray data from June (16). Although the type for V1324 Sco is currently unclear, its optical spectroscopic evolution at early times (15) did not resemble oxygen-neon novae at similar stages. We take this to indicate that it is likely a carbon-oxygen type.

The LAT data (13) for the three classical novae are discussed together with an updated analysis of the originally detected symbiotic nova V407
Table 1. Summary of the four novae. Tabulated are optical peak magnitudes and adopted distances from (19) for V407 Cyg, estimate of 4- to 5-kpc V1324 Sco based on the maximum magnitude rate of decline relation (17) [notwithstanding the large uncertainties in this method (29)], (9) for V959 Mon (scaled from V1974 Cyg 1992), and (30) for V339 Del (scaled from OS Andromedae 1986), and observed dates of the optical peaks [unfiltered from (3), V-band, adopted, and visual magnitudes, respectively]. Positions in J2000.0 equinox (right ascension, RA; declination, Decl.), Galactic longitude $(I)$ and latitude (b), 95\% confidence localization error radius, and offset between the LAT and optical positions in units of degrees. Adopted start dates $t_{\mathrm{s}}(13)$ are given in Gregorian dates and modified Julian days (MJD). The $\gamma$-ray luminosities $L_{\gamma}$ and total emitted energies were estimated with the average fluxes from the power-law fits of the $>100-\mathrm{MeV}$ LAT spectra integrated up to $10 \mathrm{GeV}$ and durations from $t_{\mathrm{s}}$ up to the last $>2 \sigma$ daily bin LAT detection. For V339 Del, the $\gamma$ rays were detected for 25 days in 1-day bins (Fig. 2), but there was a hint of a detection 2 days earlier on the day of the optical peak in 0.5-day binned data (13), leading to a 27-day duration.

\begin{tabular}{|c|c|c|c|c|}
\hline Nova & V407 Cyg 2010 & V1324 Sco 2012 & V959 Mon 2012 & V339 Del 2013 \\
\hline Distance (kpc) & 2.7 & 4.5 & 3.6 & 4.2 \\
\hline Peak magnitude & 6.9 & 10.0 & $5^{*}$ & 4.3 \\
\hline Peak date & 10.80 Mar 2010 & 19.96 Jun 2012 & - & 16.50 Aug 2013 \\
\hline Optical RA, Decl. & $315.5409^{\circ},+45.7758^{\circ}$ & $\begin{array}{l}267.7246^{\circ} \\
-32.6224^{\circ}\end{array}$ & $99.9108^{\circ},+5.8980^{\circ}$ & $305.8792^{\circ},+20.7681^{\circ}$ \\
\hline Optical I, b & $\begin{array}{l}86.9826^{\circ} \\
-0.4820^{\circ}\end{array}$ & $\begin{array}{l}357.4255^{\circ} \\
-2.8723^{\circ}\end{array}$ & $206.3406^{\circ},+0.0754^{\circ}$ & $\begin{array}{l}62.2003^{\circ} \\
-9.4234^{\circ}\end{array}$ \\
\hline LAT RA, Decl. & $315.57^{\circ},+45.75^{\circ}$ & $\begin{array}{l}267.72^{\circ} \\
-32.69^{\circ}\end{array}$ & $99.98^{\circ},+5.86^{\circ}$ & $305.91^{\circ},+20.78^{\circ}$ \\
\hline Optical-LAT offset & $0.03^{\circ}$ & $0.07^{\circ}$ & $0.08^{\circ}$ & $0.03^{\circ}$ \\
\hline LAT error radius (95\%) & $0.08^{\circ}$ & $0.09^{\circ}$ & $0.18^{\circ}$ & $0.12^{\circ}$ \\
\hline$t_{\mathrm{s}}$ (date) & 10 Mar 2010 & 15 Jun 2012 & 19 Jun 2012 & 16 Aug 2013 \\
\hline$t_{\mathrm{s}}(\mathrm{MJD})$ & 55265 & 56093 & 56097 & 56520 \\
\hline Duration (days) & 22 & 17 & 22 & 27 \\
\hline$L_{\gamma}\left(10^{35} \mathrm{erg} \mathrm{s}^{-1}\right)$ & 3.2 & 8.6 & 3.7 & 2.6 \\
\hline Total energy $\left(10^{41} \mathrm{erg}\right)$ & 6.1 & 13 & 7.1 & 6.0 \\
\hline
\end{tabular}

*For V959 Mon, the optical peak magnitude of 9.4 (unfiltered) was observed $~ 50$ days after the initial $\gamma$-ray detection, and we adopted an inferred peak of 5 magnitude (9). 
Cyg (3). The $\gamma$-ray light curves of all four systems (Fig. 2) are similar, with 2- to 3-day-long peaks occurring 3 to 5 days after the initial LAT detections. The observed optical peak preceded the $\gamma$-ray peak by $\sim 2$ days in V1324 Sco $(13,17)$ and $\sim 6$ days in V339 Del $(12,13)$. Because the early optical light variations of the ejecta in novae are driven by line opacity changes in the ultraviolet during the expansion, the rise to peak optical brightness coincides with the maximum flux redistribution toward lower energies as the optically thick surface moves outward [see (18)]. The initial lack of detected $\gamma$ rays could be because the ejecta are opaque and any $>100-\mathrm{MeV}$ emissions produced are absorbed by photon-atom interactions, with $\gamma$ rays appearing only later when the density drops and the ejecta become transparent. The three novae were detected in $\gamma$ rays during a time of high $\mathrm{x}$-ray and ultraviolet/optical opacity. Coincidentally, the few days' delay of the $\gamma$-ray peak relative to the optical peak was also observed in V407 Cyg, but this may instead signal interactions with its red giant companion (below).

In compact classical nova binaries, typical companion separations are $a \sim 10^{11} \mathrm{~cm}[\sim 100$ times

Fig. 1. Fermi-LAT $>100-M e V \gamma$-ray counts maps of the four novae in Galactic coordinates centered on the optical positions over the full 17- to 27-day durations. The maps used $0.1^{\circ}$ by $0.1^{\circ}$ pixels and were adaptively smoothed with a minimum number of 25 to 50 counts per kernel. Each nova (located at the centers of the yellow circles with $1^{\circ}$ radius, which is the approximate LAT $95 \%$ containment at $1 \mathrm{GeV}$ ) is observed near the bright diffuse $\gamma$-ray emission in the Galactic plane, with V959 Mon in particular observed directly through the plane ( $0^{\circ}$ latitude). larger in symbiotic systems (19)], and expansion velocities $v_{\mathrm{ej}}$ at early times are many $100 \mathrm{~s}$ to $\gtrsim 1000 \mathrm{~km} \mathrm{~s}^{-1}$. Thus, the ejecta reach the companion on a time scale of $t=1000\left(a / 10^{11} \mathrm{~cm}\right)$ $\left(v_{\mathrm{ej}} / 1000 \mathrm{~km} \mathrm{~s}^{-1}\right)^{-1} \mathrm{~s}$ (i.e., on the order of an hour or less). Modeling of the optical line profiles indicates that the spatial distribution of the ejected gas is bipolar rather than spherical in all cases, with greater extension perpendicular to the orbital plane in V959 Mon $(9,20,21)$. Also, narrow absorption and emission line structures seen in optical and ultraviolet line profiles later in the expansion may be evidence of hydrodynamical instabilities and multiple ejections that may lead to the formation of strong turbulence and internal shocks within the ejecta after the ignition of the thermonuclear runaway (22). A clue to the physical process that causes the $\gamma$-ray emission mechanism may be the similarity of the high-energy spectral characteristics of V1324 Sco, V959 Mon, and V339 Del. Their $>100-\mathrm{MeV}$ spectra are all soft and can be fit with single power laws [the spectrum $N(E) \propto E^{-\Gamma}$, where $N$ is the number of photons and $E$ is energy] with photon indices $\Gamma=2.1$ to 2.3 , or
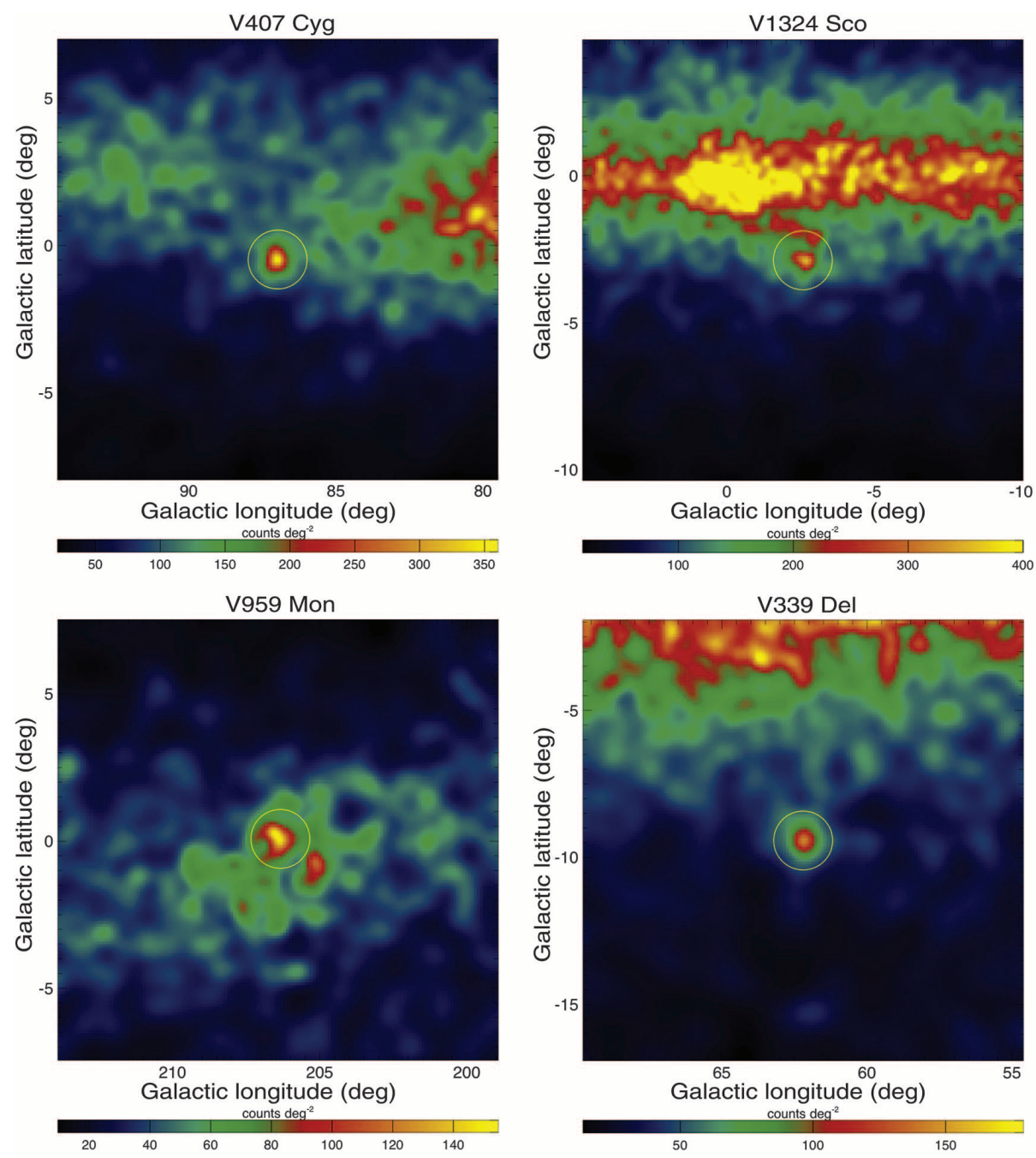

exponentially cutoff power laws [the spectrum $N(E) \propto E^{-s} e^{-E / E_{c}}$, where $E_{\mathrm{c}}$ is the cutoff energy] [see (13), table S1, and fig. S1]. The exponentially cutoff power-law fits to the LAT data were preferred over the power-law fits at the $3.8 \sigma$ and 3.4 $\sigma$ level for V959 Mon and V339 Del, respectively, but provided an insignificant improvement $(2.0 \sigma)$ for V1324 Sco. Considering the uncertainties in the spectral fits, the three novae are similarly characterized by slopes $s=$ 1.7 to $1.8, E_{\mathrm{c}} \sim 1$ to $4 \mathrm{GeV}$, and observed emission up to $\sim 6$ to $10 \mathrm{GeV}$. The total durations of the observed $\gamma$ rays were also similar, being detected for 17 to 27 days at $>2 \sigma$ statistical significances in daily bins (Fig. 2 and Table 1). Because the LATobserved properties are similar, it is likely that the $\gamma$-ray emission of these classical novae has a similar origin, involving interactions of the accelerated high-energy protons (hadronic scenario) or electrons (leptonic scenario) within the ejecta.

In the hadronic scenario, high-energy protons $\left(\pi^{0}\right)$, which decay into two $\gamma$ rays. For a representative hadronic model, we assume an exponentially cutoff power law distribution of that interact with nuclei produce neutral pions
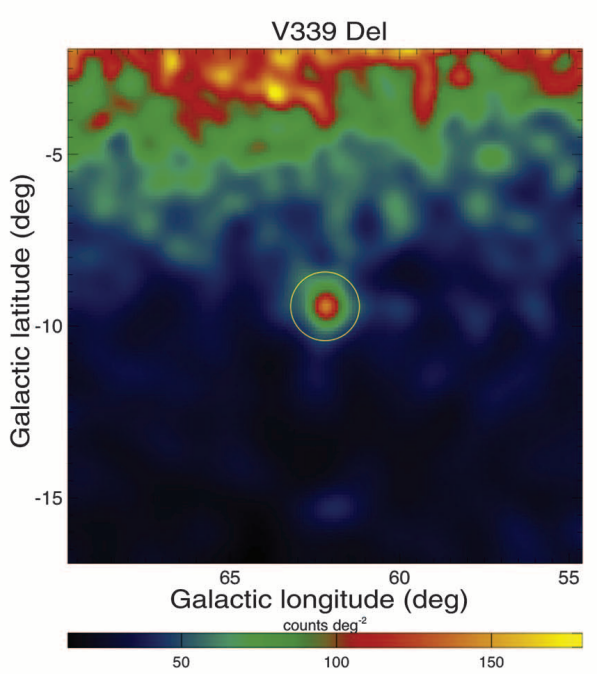
Fig. 2. Fermi-LAT 1-day binned light curves of the four $\gamma$-ray detected novae. Vertical bars indicate $1 \sigma$ uncertainties for data points with $>3 \sigma$ (black) and 2 to $3 \sigma$ (gray) significances; otherwise, $2 \sigma$ upper limits are indicated with gray arrows. Start times $t_{\mathrm{s}}$ (from top to bottom panels) of 16 August 2013, 19 June 2012, 15 June 2012, and 10 March 2010 were defined as the day of the first $\gamma$-ray detection. In V339 Del, there was a $2.4 \sigma$ detection in 0.5-day binned data beginning 16.5 August (13), the epoch of the optical peak (blue diamond in each panel).

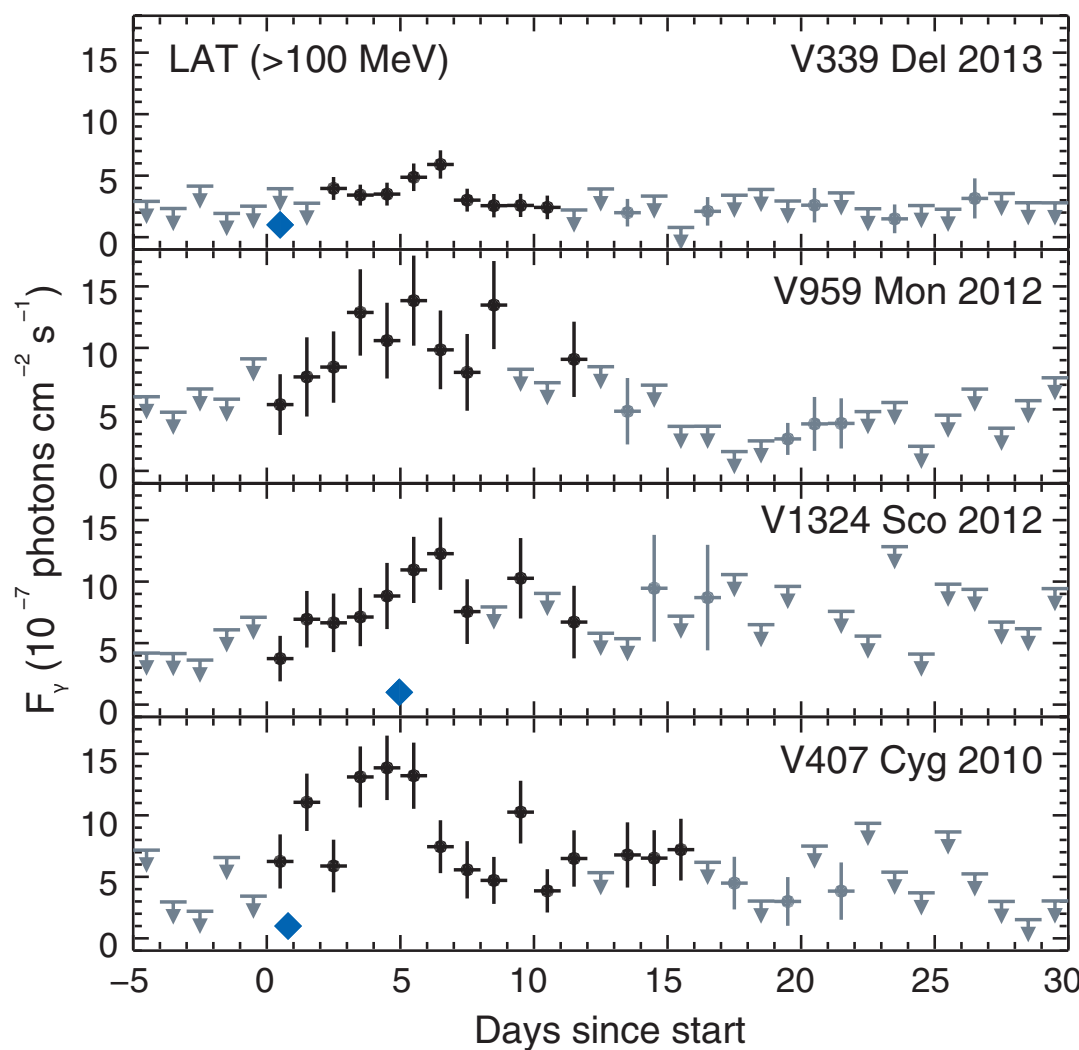

In the leptonic case, accelerated electrons produce $\gamma$ rays through a combination of inverse Compton scattering with low-energy photons and bremsstrahlung with atoms in the vicinity of the nova. For a leptonic model, we adopted a similar functional form for the distribution of the kinetic energy of high-energy electrons $\left(W_{\mathrm{e}}\right)$ in the form $N_{\mathrm{e}}\left(W_{\mathrm{e}}\right)=N_{\mathrm{e}, 0} W_{\mathrm{e}}^{-S_{\mathrm{e}}} e^{-W_{\mathrm{e}} / E_{\mathrm{ce}}}$ (electron $/ \mathrm{GeV}$ ) and fitted the normalization $N_{\mathrm{e}, \mathrm{o}}$, slope $s_{\mathrm{e}}$, and cutoff energy $E_{\mathrm{ce}}$ to the LAT data for each nova (Fig. 3). The $\gamma$-ray luminosity of the calculated bremsstrahlung emission is $<20 \%$ of the total $\gamma$-ray luminosity for all the novae (13). The best-fit parameters of the high-energy electron spectra for the three classical novae are similar within their confidence regions (13), with $E_{\text {ce }}$ constrained to lie between 2 and $30 \mathrm{GeV}$ and poorly constrained slopes. These models are statistically indistinguishable from the $\pi^{0}$ model. As in the hadronic model, the spectral parameters of the classical novae differ from those for V407 Cyg (mainly due to the lowestenergy $\sim 200$ - to $300-\mathrm{MeV}$ bin detected in its LAT spectrum), where the best-fit slope is negative (i.e., a positive index of the power law) and $E_{\text {ce }}=$ $1.78 \pm 0.05 \mathrm{GeV}$. The best-fit parameters for the leptonic scenario, where high-energy electrons interact primarily with the photons emitted by the nova photosphere (23), lead to total energies of $\sim(6$ to 13$) \times 10^{41} \mathrm{ergs}$ in high-energy electrons and conversion efficiencies of $\sim 0.1$ to $0.3 \%$ for the classical novae and $0.6 \%$ for the symbiotic system.

Detection of classical novae in $\gamma$ rays was deemed unlikely in the past (3). The only nova previously detected in $\gamma$ rays, the aforementioned V407 Cyg, was a rare symbiotic and likely recurrent [only 10 recurrent novae are known, of which 4 are symbiotic types (24)]. In the symbiotic novae, conditions are conducive for highenergy particle acceleration as the portion of the ejecta moving into the wind in the direction of the dense medium provided by the red giant companion decelerates within a few days. The $\gamma$ rays peak early, when the efficiency for hadron and lepton acceleration is presumably favorable, with the red giant wind playing a key role in the $\gamma$-ray production $(2,23)$. In contrast, the mainsequence star companions in the classical novae do not provide similarly dense target material; hence, it is likely that other dissipative processes are involved in particle acceleration and generation of the observed $\gamma$ rays.

Because the $\gamma$-ray properties of the novae detected so far by the Fermi-LAT appear similar to one another, and their underlying properties are unremarkable, it appears that all novae can be considered to be candidate $\gamma$-ray emitters. Their detection by the LAT may imply close proximity and that other optical novae not yet detected with the LAT [e.g., (25)] are more distant and have fainter optical peaks [without considering extinction uncertainties (26)]. Indeed, all the LAT-detected novae have estimated distances of $\lesssim 4$ to $5 \mathrm{kpc}$ (Table 1). Despite systematic uncertainties in the adopted distances, it is interesting that the inferred mean $\gamma$-ray luminosities and total emitted energies of the novae span a small range $\sim(3$ to 4$) \times 10^{35} \mathrm{ergs} \mathrm{s}^{-1}$ and $\sim(6$ to 7$) \times$ $10^{41}$ ergs, respectively, except for the $\sim 2$ times $\sim 0.1$ to $3.7 \%$ for the classical novae and $6.6 \%$ for V407 Cyg. 

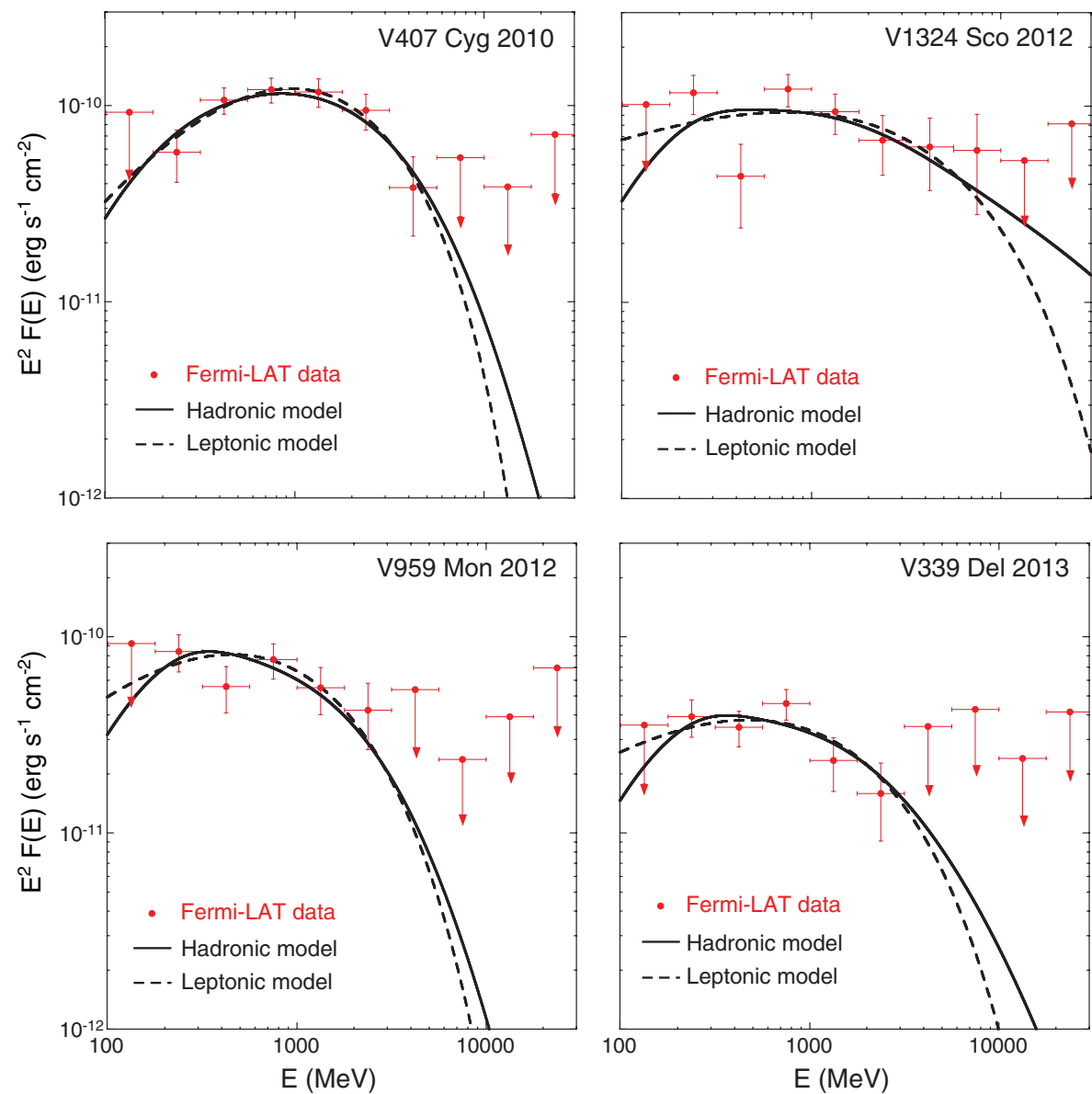

Fig. 3. Fermi-LAT $>100-\mathrm{MeV}$ average $\gamma$-ray spectra of the four novae over the full 17- to 27 -day durations. Vertical bars indicate $1 \sigma$ uncertainties for data points with significances $>2 \sigma$ otherwise, arrows indicate $2 \sigma$ limits. The best-fit hadronic and leptonic model curves are overlaid.

greater values for V1324 Sco, whose distance is highly uncertain.

The rate of novae in the Milky Way is highly uncertain, but considering a plausible range of $\sim 20$ to 50 per year (27) and reasonable spatial distributions in the Galactic bulge and disk (28), our estimate is 1 to 4 per year at $\lesssim 4$ - to 5 -kpc distances. The $\gamma$-ray detection rate of novae averages roughly one per year over the time span of these observations ( $\sim 5$ years), consistent with the lower end of this extrapolation.

Although the $\gamma$-ray properties of the LATdetected novae are similar, we emphasize the small and subtle differences that imply different emission mechanisms-e.g., the spectral shape of V407 Cyg compared with the three classical novae as well as the apparent higher energy extension of the V1324 Sco spectrum. Among the classical novae detected so far, they also appear different optically. The $\gamma$-ray emission mechanism and high-energy particle acceleration processes associated with the novae could depend on the particular system properties that remain to be investigated, such as the white dwarf mass, which determines the explosion energetics (ejected mass and expansion velocity), and the mass transfer dictated by the companion mass and separation.

\section{REFERENCES AND NOTES}

1. W. B. Atwood et al., Astrophys. J. 697, 1071-1102 (2009)

2. V. Tatischeff, M. Hernanz, Astrophys. J. 663, L101-L104 (2007).

3. A. A. Abdo et al., Science 329, 817-821 (2010).

4. V. L. Ginzburg, S. I. Syrovatskii, The Origin of Cosmic Rays (Macmillan, New York, 1964), pp. 200-202.

5. M. Hernanz, in Classical Novae, M. F. Bode, A. Evans, Eds. (Cambridge Univ. Press, Cambridge, ed. 2, 2008) p. 252.

6. D. Takei et al., Astrophys. J. 697, L54-L57 (2009)

7. C. C. Cheung, E. Hays, T. Venters, D. Donato, R. H. D. Corbet, Fermi-LAT Collaboration, The Astronomer's Telegram 4224 1 (2012).

8. S. Fujikawa, International Astronomical Union Central Bureau for Astronomical Telegrams, reported by M. Soma, no. 3202 (2012).

9. S. N. Shore et al., Astron. Astrophys. 553, A123 (2013).

10. S. Starrfield, W. M. Sparks, J. W. Truran, Astrophys. J. 303, L5 (1986).

11. K. Itagaki, International Astronomical Union Central Bureau for Astronomical Telegrams, reported by S. Nakano, no. 3628 (2013).

12. E. Hays, T. Cheung, S. Ciprini; Fermi-LAT collaboration, The Astronomer's Telegram 5302, 1 (2013).

13. Supplementary materials are available on Science Online. 5282, 1 (2013)

15. R. M. Wagner et al., The Astronomer's Telegram 4157, 1 (2012).

16. C. C. Cheung, T. Glanzman, A. B. Hill, Fermi-LAT Collaboration, The Astronomer's Telegram 4284, 1 (2012).

17. A. B. Hill, Fermi-LAT collaboration, in 4th Fermi Symposium, Monterey CA, eConf C121028, 112 (2012), 28 October to 2 November 2012.
14. S. N. Shore, P. Skoda, P. Rutsch, The Astronomer's Telegram
18. S. N. Shore, Bull. Astron. Soc. India 40, 185-191 (2012).

19. U. Munari, R. Margoni, R. Stagni, Mon. Not. R. Astron. Soc. 242 653 (1990).

20. V. A. R. M. Ribeiro, U. Munari, P. Valisa, Astrophys. J. 768, 49 (2013).

21. G. H. Herbig, J. I. Smak, Acta Astronomica. 42, 17-28 (1992)

22. J. Casanova, J. José, E. García-Berro, S. N. Shore, A. C. Calder Nature 478, 490-492 (2011).

23. P. Martin, G. Dubus, Astron. Astrophys. 551, A37 (2013).

24. B. E. Schaefer, Astrophys. J. Suppl. Ser. 187, 275-373 (2010).

25. C. C. Cheung, Fermi-LAT collaboration, in 4th Fermi Symposium, Monterey CA, eConf C121028, 106 (2012), 28 October to 2 November 2012.

26. K. Mukai, http://asd.gsfc.nasa.gov/Koji.Mukai/novae/novae. html, September 4, 2013 version.

27. A. W. Shafter, Astrophys. J. 487, 226-236 (1997).

28. P. Jean, M. Hernanz, J. Gómez-Gomar, J. José, Mon. Not. R. Astron. Soc. 319, 350-364 (2000).

29. M. M. Kasliwal et al., Astrophys. J. 735, 94 (2011).

30. S. N. Shore, The Astronomer's Telegram 5410, 1 (2013).

\section{ACKNOWLEDGMENTS}

The Fermi-LAT Collaboration acknowledges support for LAT development, operation, and data analysis from NASA and the Department of Energy (United States), CEA/Irfu and IN2P3/CNRS (France), Agenzia Spaziale Italiana and INFN (Italy), MEXT, KEK, and JAXA (Japan), and the K. A. Wallenberg Foundation, the Swedish Research Council, and the National Space Board (Sweden). Science analysis support in the operations phase from INAF (Italy) and CNES (France) is also gratefully acknowledged. We acknowledge with thanks the variable star observations from the American Association of Variable Star Observers International Database contributed by observers worldwide and used in this research and the dedicated observers of the Astronomical Ring for Access to Spectroscopy (ARAS) group for their tireless and selfless efforts. C.C.C. was supported at the Naval Research Laboratory by a Karles' Fellowship and by NASA through DPR S-15633-Y and Guest Investigator programs 11-FERMI11-0030 and 12-FERMI12-0026. S.S. was supported by NASA and NSF grants to Arizona State University. The Fermi-LAT data reported in this paper are available from http://fermi.gsfc.nasa.gov/ssc/data/access.

\section{The Fermi-LAT Collaboration}

M. Ackermann, ${ }^{1}$ M. Ajello, ${ }^{2}$ A. Albert, ${ }^{3}$ L. Baldini, ${ }^{4}$ J. Ballet, ${ }^{5}$ G. Barbiellini, ${ }^{6,7}$ D. Bastieri, ${ }^{8,9}$ R. Bellazzini, ${ }^{4}$ E. Bissaldi, ${ }^{10}$ R. D. Blandford ${ }^{3}$ E. D. Bloom ${ }^{3}$ E. Bottacini, ${ }^{3}$ T. J. Brandt, ${ }^{11}$ J. Bregeon, ${ }^{12}$ P. Bruel, ${ }^{13}$ R. Buehler, ${ }^{1}$ S. Buson, ${ }^{8,9}$ G. A. Caliandro, ${ }^{3,14}$ R. A. Cameron, ${ }^{3}$ M. Caragiulo, ${ }^{15}$ P. A. Caraveo, ${ }^{16}$ E. Cavazzuti, ${ }^{17}$ E. Charles, ${ }^{3}$ A. Chekhtman, ${ }^{18 \ddagger}$ C. C. Cheung, ${ }^{19+}$ J. Chiang, ${ }^{3}$ G. Chiaro, ${ }^{9}$ S. Ciprini, ${ }^{17,20}$ R. Claus, ${ }^{3}$ J. Cohen-Tanugi, ${ }^{12}$ J. Conrad, ${ }^{21,22,23,24}$ S. Corbel, ${ }^{5,25}$ F. D'Ammando, ${ }^{26,27}$ A. de Angelis, ${ }^{28}$ P. R. den Hartog, ${ }^{3}$ F. de Palma, ${ }_{15}$ C. D. Dermer, ${ }^{19}$ R. Desiante, ${ }^{6,29}$ S. W. Digel, ${ }^{3}$ L. Di Venere, ${ }^{30}$ E. do Couto e Silva, ${ }^{3}$ D. Donato, ${ }^{31,32}$ P. S. Drell, ${ }^{3}$ A. Drlica-Wagner, ${ }^{33}$ C. Favuzzi, ${ }^{30,15}$ E. C. Ferrara, ${ }^{11}$ W. B. Focke, ${ }^{3}$ A. Franckowiak, ${ }^{3}$ L. Fuhrmann, ${ }^{34}$ Y. Fukazawa, ${ }^{35}$ P. Fusco, ${ }^{30,15}$ F. Gargano, ${ }^{15}$ D. Gasparrini, ${ }^{17,20}$ S. Germani, ${ }^{36,37}$ N. Giglietto, ${ }^{30,15}$ F. Giordano, ${ }^{30,15}$ M. Giroletti, ${ }^{26}$ T. Glanzman, ${ }^{3}$ G. Godfrey, ${ }^{3}$ I. A. Grenier, ${ }^{5}$ J. E. Grove, ${ }^{19}$ S. Guiriec, ${ }^{11,38}$ D. Hadasch, ${ }^{39}$ A. K. Harding, ${ }^{11}$ M. Hayashida, ${ }^{40}$ E. Hays, ${ }^{11}$ J. W. Hewitt, ${ }^{41,31}$ A. B. Hill, ${ }^{42,3,43}$ X. Hou, ${ }^{44}$ P. Jean, ${ }^{45,46 \dagger}$ T. Jogler, ${ }^{3}$ G. Jóhannesson, ${ }^{47}$ A. S. Johnson, ${ }^{3}$ W. N. Johnson, ${ }^{19}$ M. Kerr, ${ }^{48}$ J. Knödlseder, ${ }^{45,46}$ M. Kuss, ${ }^{4}$ S. Larsson, ${ }^{21,22,49}$ L. Latronico, ${ }^{50}$ M. Lemoine-Goumard, ${ }^{44,51}$ F. Longo, ${ }^{6,7}$ F. Loparco, ${ }^{30,15}$ B. Lott, ${ }^{44}$ M. N. Lovellette, ${ }^{19}$ P. Lubrano, ${ }^{36,37}$ A. Manfreda, ${ }^{4}$ P. Martin, ${ }^{46}$ F. Massaro, ${ }^{52}$ M. Mayer, ${ }^{1}$ M. N. Mazziotta, ${ }^{15}$ J. E. McEnery, ${ }^{11,32}$ P. F. Michelson, ${ }^{3}$ W. Mitthumsiri, ${ }^{53,3}$ T. Mizuno, ${ }^{54}$ M. E. Monzani, ${ }^{3}$ A. Morselli, ${ }^{55}$ I. V. Moskalenko, ${ }^{3}$ S. Murgia, ${ }^{56}$ R. Nemmen, ${ }^{11,31,41}$ E. Nuss, ${ }^{12}$ T. Ohsugi, ${ }^{54}$ N. Omodei, ${ }^{3}$ M. Orienti, ${ }^{26}$ E. Orlando, ${ }^{3}$ J. F. Ormes, ${ }^{57}$ D. Paneque, ${ }^{58,3}$ J. H. Panetta, ${ }^{3}$ J. S. Perkins, ${ }^{11}$ M. Pesce-Rollins, ${ }^{4}$ F. Piron, ${ }^{12}$ G. Pivato, ${ }^{9}$ T. A. Porter, ${ }^{3}$ S. Rainò, ${ }^{30,15}$ R. Rando, ${ }^{8,9}$ M. Razzano, ${ }^{4,59}$ S. Razzaque, A. Reimer, ${ }^{39,3}$ O. Reimer, ${ }^{39,3}$ T. Reposeur, ${ }^{44}$ P. M. Saz Parkinson, ${ }^{61,62}$ M. Schaal, ${ }^{6 \ddagger}$ A. Schulz, ${ }^{1}$ C. Sgrò, ${ }^{4}$ E. J. Siskind, ${ }^{64}$ G. Spandre, ${ }^{4}$ P. Spinelli, ${ }^{30,15}$ Ł. Stawarz, ${ }^{65,66}$ D. J. Suson, ${ }^{67}$ H. Takahashi, ${ }^{35}$ T. Tanaka, ${ }^{68}$ J. G. Thayer, ${ }^{3}$ J. B. Thayer, ${ }^{3}$ D. J. Thompson, ${ }^{11}$ L. Tibaldo, ${ }^{3}$ M. Tinivella, ${ }^{4}$ D. F. Torres, ${ }^{69,70}$ G. Tosti, ${ }^{36,37}$ E. Troja, ${ }^{11,32}$ Y. Uchiyama, ${ }^{71}$ G. Vianello, ${ }^{3}$ B. L. Winere, ${ }^{72}$ M. T. Wolff, ${ }^{19}$ D. L. Wood, ${ }^{7 \ddagger}$ K. S. Wood, ${ }^{19}$ M. Wood, ${ }^{3}$ S. Charbonnel, ${ }^{74}$ R. H. D. Corbet, ${ }^{31,41}$ I. De Gennaro Aquino, ${ }^{75,76}$ J. P. Edlin, ${ }^{77}$ E. Mason, ${ }^{78}$ G. J. Schwarz, ${ }^{79}$ S. N. Shore, ${ }^{4,75+}$ S. Starrfield, ${ }^{80}$ F. Teyssier ${ }^{81}$

${ }^{1}$ Deutsches Elektronen Synchrotron DESY, D-15738 Zeuthen, Germany. ${ }^{2}$ Department of Physics and Astronomy, Clemson University, Kinard Lab of Physics, Clemson, SC 29634-0978, USA. ${ }^{3}$ W. W. Hansen 
Experimental Physics Laboratory, Kavli Institute for Particle Astrophysics and Cosmology, Department of Physics and SLAC National Accelerator Laboratory, Stanford University, Stanford, CA 94305, USA. ${ }^{4}$ Istituto Nazionale di Fisica Nucleare, Sezione di Pisa, I-56127 Pisa, Italy. ${ }^{5}$ Laboratoire AIM, CEA-IRFU/CNRS/Université Paris Diderot, Service d'Astrophysique, CEA Saclay, 91191 Gif sur Yvette, France. ${ }^{6}$ Istituto Nazionale di Fisica Nucleare, Sezione di Trieste 1-34127 Trieste, Italy. ${ }^{7}$ Dipartimento di Fisica, Università di Trieste, I34127 Trieste, Italy. ${ }^{8}$ Istituto Nazionale di Fisica Nucleare, Sezione di Padova, I-35131 Padova, Italy. ${ }^{9}$ Dipartimento di Fisica e Astronomia "G. Galilei", Università di Padova, I-35131 Padova, Italy. ${ }^{10}$ Istituto Nazionale di Fisica Nucleare, Sezione di Trieste, and Università di Trieste, I-34127 Trieste, Italy. ${ }^{11}$ NASA Goddard Space Flight Center, Greenbelt, MD 20771, USA. ${ }^{12}$ Laboratoire Univers et Particules de Montpellier, Université Montpellier 2, CNRS/IN2P3, Montpellier, France. ${ }^{13}$ Laboratoire Leprince-Ringuet, École polytechnique, CNRS/IN2P3, Palaiseau, France. ${ }^{14}$ Consorzio Interuniversitario per la Fisica Spaziale (CIFS), I-10133 Torino, Italy. ${ }^{15}$ Istituto Nazionale di Fisica Nucleare. Sezione di Bari, 70126 Bari, Italy. ${ }^{16}$ INAF-Istituto di Astrofisica Spaziale e Fisica Cosmica, I-20133 Milano, Italy. ${ }^{17}$ Agenzia Spaziale Italiana (ASI) Science Data Center, I-00133 Roma, Italy. ${ }^{18}$ Center for Earth Observing and Space Research, College of Science, George Mason University, Fairfax, VA 22030, USA. ${ }^{19}$ Space Science Division, Naval Research Laboratory, Washington, DC 20375-5352, USA. ${ }^{20}$ Istituto Nazionale di Astrofisica, Osservatorio Astronomico di Roma, 1-00040 Monte Porzio Catone (Roma), Italy. ${ }^{21}$ Department of Physics, Stockholm University, AlbaNova, SE-106 91 Stockholm, Sweden. ${ }^{22}$ The Oskar Klein Centre fo Cosmoparticle Physics, AlbaNova, SE-106 91 Stockholm, Sweden. ${ }^{23}$ Royal Swedish Academy of Sciences Research Fellow, funded by a grant from the K. A. Wallenberg Foundation. ${ }^{24}$ The Royal Swedish Academy of Sciences, Box 50005, SE-104 05 Stockholm. Sweden. ${ }^{25}$ Institut Universitaire de France, 75005 Paris, France. ${ }^{26}$ INAF Istituto di Radioastronomia, 40129 Bologna, Italy. ${ }^{27}$ Dipartimento di Astronomia, Università di Bologna, I-40127 Bologna, Italy. ${ }^{28}$ Dipartimento di Fisica, Università di Udine and Istituto Nazionale di Fisica Nucleare Sezione di Trieste, Gruppo Collegato di Udine, I-33100 Udine. ${ }^{29}$ Università di Udine, I-33100 Udine, Italy. ${ }^{30}$ Dipartimento di Fisica “M. Merlin" dell'Università e del Politecnico di Bari, I-70126 Bari, Italy. ${ }^{31}$ Center for Research and Exploration in Space Science and Technology (CRESST) and NASA Goddard Space Flight Center, Greenbelt, MD 20771, USA. ${ }^{32}$ Department of Physics and Department of Astronomy, University of Maryland, College Park, MD 20742. USA. ${ }^{33}$ Fermilab, Batavia, IL 60510, USA. ${ }^{34}$ Max-Planck-Institut für Radioastronomie, Auf dem Hügel 69, 53121 Bonn, Germany. ${ }^{35}$ Department of Physical Sciences, Hiroshima University, HigashiHiroshima, Hiroshima 739-8526, Japan. ${ }^{36}$ Istituto Nazionale di Fisica Nucleare, Sezione di Perugia, I-06123 Perugia, Italy. ${ }^{37}$ Dipartimento di Fisica, Università degli Studi di Perugia, I-06123 Perugia, Italy. ${ }^{38}$ NASA Postdoctoral Program Fellow, USA. ${ }^{39}$ Institut für Astround Teilchenphysik and Institut für Theoretische Physik, LeopoldFranzens-Universität Innsbruck, A-6020 Innsbruck, Austria. ${ }^{40}$ Institute for Cosmic-Ray Research, University of Tokyo, 5-1-5 Kashiwanoha, Kashiwa, Chiba, 277-8582, Japan. ${ }^{41}$ Department of Physics and Center for Space Sciences and Technology, University of Maryland Baltimore County, Baltimore, MD 21250, USA. ${ }^{42}$ School of Physics and Astronomy, University of Southampton, Highfield, Southampton S017 1BJ, UK. ${ }^{43}$ Funded by a Marie Curie IOF, FP7/2007-2013 grant agreement no. $275861 .{ }^{44}$ Centre d'Études Nucléaires de Bordeaux Gradignan, IN2P3/CNRS, Université Bordeaux 1, BP120, F-33175 Gradignan Cedex, France. ${ }^{45}$ CNRS, IRAP, F-31028 Toulouse cedex 4, France. ${ }^{46}$ GAHEC, Université de Toulouse, UPS-OMP, IRAP, Toulouse, France. ${ }^{47}$ Science Institute, University of Iceland, IS-107 Reykjavik, Iceland. ${ }^{48} \mathrm{CSIRO}$ Astronomy and Space Science, Australia Telescope National Facility, Epping NSW 1710, Australia. ${ }^{49}$ Department of Astronomy, Stockholm University, SE-106 91 Stockholm, Sweden. ${ }^{50}$ Istituto Nazionale di Fisica Nucleare, Sezione di Torino, I-10125 Torino, Italy. ${ }^{51}$ Funded by contract ERC-StG-259391 from the European Community. ${ }^{52}$ Department of Astronomy, Department of Physics, and Yale Center for Astronomy and Astrophysics, Yale University, New Haven, CT 06520-8120, USA. ${ }^{53}$ Department of Physics, Faculty of Science, Mahidol University, Bangkok 10400, Thailand. ${ }^{54}$ Hiroshima Astrophysical Science Center, Hiroshima University, Higashi-Hiroshima, Hiroshima 739-8526, Japan. ${ }^{55}$ Istituto Nazionale di Fisica Nucleare, Sezione di Roma "Tor Vergata", I-00133 Roma, Italy. ${ }^{56}$ Center for Cosmology, Physics and Astronomy Department, University of California, Irvine, CA 92697-2575, USA. ${ }^{57}$ Department of Physics and Astronomy, University of Denver, Denver, CO 80208, USA. ${ }^{58}$ Max-Planck-Institut für Physik, D-80805 München, Germany. ${ }^{59}$ Funded by contract FIRB-2012-RBFR12PM1F from the Italian Ministry of Education, University and Research (MIUR) ${ }^{60}$ Department of Physics, University of Johannesburg, P.O. Box 524, Auckland Park 2006, South Africa. ${ }^{61}$ Santa Cruz Institute for Particle Physics, Department of Physics and Department of Astronomy and
Astrophysics, University of California at Santa Cruz, Santa Cruz, CA 95064, USA. ${ }^{62}$ Department of Physics, The University of Hong Kong, Pokfulam Road, Hong Kong. China. ${ }^{63}$ National Research Council Research Associate, National Academy of Sciences, Washington, DC 20001, USA.. ${ }^{64}$ NYCB Real-Time Computing Inc., Lattingtown, NY 11560-1025, USA. ${ }^{65}$ Institute of Space and Astronautical Science, Japan Aerospace Exploration Agency, 3-1-1 Yoshinodai, Chuo-ku, Sagamihara, Kanagawa 252-5210, Japan. ${ }^{66}$ Astronomical Observatory, Jagiellonian University, 30-244 Kraków, Poland. ${ }^{67}$ Department of Chemistry and Physics, Purdue University Calumet, Hammond, IN 46323-2094, USA. ${ }^{68}$ Department of Physics, Graduate School of Science, Kyoto University, Kyoto, Japan. ${ }^{69}$ Institut de Ciències de I'Espai (IEEE-CSIC), Campus UAB, 08193 Barcelona, Spain. ${ }^{70}$ Institució Catalana de Recerca i Estudis Avançats (ICREA), Barcelona, Spain. ${ }^{71} 3-34-1$ Nishi-lkebukuro, Toshima-ku, Tokyo 171-8501, Japan. ${ }^{72}$ Department of Physics, Center for Cosmology and Astro-Particle Physics. The Ohio State University, Columbus, $\mathrm{OH} 43210$, USA. ${ }^{73}$ Praxis Inc., Alexandria, VA 22303, USA. ${ }^{74}$ Durtal Observatory, 6 Rue des Glycines, F-49430 Durtal, France. ${ }^{75}$ Dipartimento di Fisica "Enrico Fermi", Università di Pisa, Pisa I-56127, Italy. ${ }^{76}$ Hamburger Sternwarte, Gojenbergs- weg 112, 21029, Hamburg, Germany. ${ }^{77}$ Ammon, ID 83401, USA ${ }^{78}$ INAF Osservatorio Astronomico di Trieste, Via G. B. Tiepolo 11 34131 Trieste, Italy. ${ }^{79}$ American Astronomical Society, 2000 Florida Ave NW, Washington, DC 20009-1231, USA. ${ }^{80}$ School of Earth and Space Exploration, Arizona State University, P.0. Box 871404, Tempe, AZ 85287-1404, USA. ${ }^{81} 67$ Rue Jacques Daviel, Rouen 76100, France. łResident at Naval Research Laboratory, Washington, DC 20375, USA

\section{SUPPLEMENTARY MATERIALS}

www.sciencemag.org/content/345/6196/554/suppl/DC1

Materials and Methods

Supplementary Text

Figs. S1 to S6

Tables S1 to S4

References (31-48)

26 March 2014; accepted 20 June 2014

$10.1126 /$ science. 1253947

\section{A network framework of cultural history}

\section{Maximilian Schich, ${ }^{1,2,3 *}$ Chaoming Song, ${ }^{4}$ Yong-Yeol Ahn, ${ }^{5}$ Alexander Mirsky, ${ }^{2}$ Mauro Martino, ${ }^{3}$ Albert-László Barabási, ${ }^{3,6,7}$ Dirk Helbing ${ }^{2}$}

The emergent processes driving cultural history are a product of complex interactions among large numbers of individuals, determined by difficult-to-quantify historical conditions. To characterize these processes, we have reconstructed aggregate intellectual mobility over two millennia through the birth and death locations of more than 150,000 notable individuals. The tools of network and complexity theory were then used to identify characteristic statistical patterns and determine the cultural and historical relevance of deviations. The resulting network of locations provides a macroscopic perspective of cultural history, which helps us to retrace cultural narratives of Europe and North America using large-scale visualization and quantitative dynamical tools and to derive historical trends of cultural centers beyond the scope of specific events or narrow time intervals.

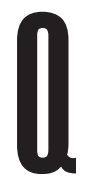

uantifying historical developments is crucial to understanding a large variety of complex processes from population dynamics to disease spreading, conflicts, and urban evolution. However, in historical research there is an inherent tension $(1,2)$ between qualitative analyses of individual historical accounts and quantitative approaches aiming to measure and model more general patterns. We believe that these approaches are complementary: We need quantitative methods to identify statistical regularities, as well as qualitative approaches to

${ }^{1}$ School of Arts and Humanities, The University of Texas at Dallas, Richardson, TX 75080, USA. ${ }^{2}$ Chair of Sociology, in particular of Modeling and Simulation (SOMS),

Eidgenössische Technische Hochschule (ETH) Zurich, CH-8092 Zurich, Switzerland. ${ }^{3}$ Center for Complex Network Research, Department of Physics, Biology and Computer Science, Northeastern University, Boston, MA 02115, USA. ${ }^{4}$ Department of Physics, University of Miami Coral Gables, Coral Gables, FL 33146, USA. ${ }^{5}$ School for Informatics and Computing, Indiana University Bloomington, Bloomington, IN 47405, USA. 'Department of Medicine, Harvard Medical School, and Center for Cancer Systems Biology, Dana-Farber Cancer Institute, Boston, MA 02115, USA. 'Center for Network Science, Central European University, Budapest 1052, Hungary

*Corresponding author. E-mail: maximilian.schich@utdallas.edu explain the impact of local deviations from the uncovered general patterns. We have therefore developed a data-driven macroscopic perspective that offers a combination of both approaches.

We collected data from Freebase.com (FB) (3), the General Artist Lexicon (AKL) (4-6), and the Getty Union List of Artist Names (ULAN) (7), representing spatiotemporal birth and death information of notable individuals, spanning a time period of more than two millennia. The data sets are included in the supplementary materials (SM), accompanied by an explanation of their nature and data preparation (8) (tables S1 and S2). Potential sources of bias are addressed in the SM, including biographical, temporal, and spatial coverage; curated versus crowd-sourced data; increasing numbers of individuals who are still alive; place aggregation; location name changes and spelling variants; and effects of data set language. Most important, compared with contemporary worldwide migration flux (9), our data sets focus on birth-to-death migration within and out of Europe and North America (see fig. S1). Notability of individuals, simply defined as the curatorial decision of inclusion in the respective data set, differs slightly between the more 
If you wish to distribute this article to others, you can order high-quality copies for your colleagues, clients, or customers by clicking here.

Permission to republish or repurpose articles or portions of articles can be obtained by following the guidelines here.

The following resources related to this article are available online at www.sciencemag.org (this information is current as of February 2, 2015 ):

Updated information and services, including high-resolution figures, can be found in the online version of this article at:

http://www.sciencemag.org/content/345/6196/554.full.html

Supporting Online Material can be found at:

http://www.sciencemag.org/content/suppl/2014/07/30/345.6196.554.DC1.html

A list of selected additional articles on the Science Web sites related to this article can be found at:

http://www.sciencemag.org/content/345/6196/554.full.html\#related

This article cites $\mathbf{4 0}$ articles, 3 of which can be accessed free:

http://www.sciencemag.org/content/345/6196/554.full.html\#ref-list-1

This article has been cited by 1 articles hosted by HighWire Press; see:

http://www.sciencemag.org/content/345/6196/554.full.html\#related-urls

This article appears in the following subject collections:

Astronomy

http://www.sciencemag.org/cgi/collection/astronomy 